\title{
STABLE UNIT BALLS IN ORLICZ SPACES
}

\author{
ANTONIO SUÁREZ GRANERO
}

(Communicated by William J. Davis)

\begin{abstract}
Let $L^{\phi}(\mu)$ be an Orlicz space and $X \subseteq L^{\phi}(\mu)$ an ideal such that $I_{\phi}(f /\|f\|)=1$ for each $f \in X \backslash\{0\}$. Then the unit ball $B_{X}$ is stable, that is, the midpoint map $\Phi_{1 / 2}: B_{X} \times B_{X} \rightarrow B_{X}$ defined by $\Phi_{1 / 2}(x, y)=\frac{1}{2}(x+y)$, is open. In particular, $B_{E} \phi$ is stable, $E^{\phi}$ being the subspace of finite elements of $L^{\phi}(\mu)$ (i.e., $f \in E^{\phi}$ iff $I_{\phi}(\lambda f)<+\infty$ for each $\left.\lambda>0\right)$, and $B_{L^{\phi}(\mu)}$ is stable when $\phi$ satisfies condition $\left(\Delta_{2}\right)$ or $\left(\delta_{2}\right)$, depending on the measure $\mu$.
\end{abstract}

If $X$ is a Banach space, we put $B_{X}$ and $S_{X}$ for the closed unit ball and unit sphere of $X$, respectively. If $x \in X$ and $\varepsilon>0$, then $B(x ; \varepsilon)=\{y \in$ $X:\|x-y\| \leq \varepsilon\}$. For a set $C$, denote by Ext. $C$, the set of its extreme points. A convex subset $C$ of a topological vector space $E$ is said stable if the midpoint map $\Phi_{1 / 2}: C \times C \rightarrow C$ such that $\Phi_{1 / 2}(x, y)=\frac{1}{2}(x+y)$, is open. Compact stable sets have been studied in $[2,8]$. Many Banach spaces have stable unit ball, namely, Banach spaces with 3.2.I.P. [2, p. 195] and finite dimensional Musielak-Orlicz spaces [3]. A Banach space $X$ with stable unit ball has interesting properties, for example:

(i) The set Ext. $B_{X}$ is closed.

(ii) If $K$ is a compact set and $C(K, X)$ denotes the Banach space of continuous functions $f: K \rightarrow X$, then $f \in$ Ext. $B_{C(K, X)}$ iff $f(k) \in$ Ext. $B_{X}$, for each $k \in K$.

In [4] Grzaslewicz proved that Orlicz spaces $L^{\phi}(\mu)$, when $\phi$ fulfills good conditions $\left(\right.$ as $\left(\Delta_{2}\right)$ or $\left.\left(\delta_{2}\right)\right)$, satisfy (ii). Here this result and others follow from the fact that the unit ball $B_{L} \phi_{(\mu)}$ of the Orlicz space $L^{\phi}(\mu)$ is stable when $\phi$ is $\left(\Delta_{2}\right)$ or $\left(\delta_{2}\right)$, depending on $\mu$.

We consider a positive measure space $(\Omega, \Sigma, \mu)$ and an Orlicz function $\phi: \mathbf{R} \rightarrow[0, \infty)$ convex, even, $\phi(0)=0$ and $\phi(x)>0$ for some $x \in \mathbf{R}$. The Orlicz space $L^{\phi}(\mu)$ is the set of (equivalence classes of) measurable functions

Received by the editors November 17, 1988.

1980 Mathematics Subject Classification (1985 Revision). Primary 46E30; Secondary 46B20, 47D20.

Key words and phrases. Stable sets, Orlicz spaces, unit ball.

Supported in part by DGICYT Grant PB88-0141. 
$f: \Omega \rightarrow \mathbf{R}$ such that the functional $I_{\phi}(\lambda f)=\int_{\Omega} \phi(\lambda f) d \mu<+\infty$, for some $\lambda>0$, equipped with Luxemburg norm $\|f\|=\inf \left\{\lambda>0: I_{\phi}(f / \lambda) \leq 1\right\}$.

The function $\phi$ satisfies condition $\left(\Delta_{2}\right)$ if there are two constants $b>0, k>$ 0 such that $\phi(2 u) \leq k \phi(u),|u| \geq b$. The function $\phi$ satisfies condition $\left(\delta_{2}\right)$ if there are two constants $a>0, k>0$ such that $\phi(a)>0$ and $\phi(2 u) \leq k \phi(u)$, $|u| \leq a$.

Let $U_{\phi}$ be the maximal open subset of $\mathbf{R}$ such that $\phi$ is affine on each connected component of $U_{\phi}$. The set $U_{\phi}$ consists of a countable (finite or infinite) number of connected components. In general, if $\omega$ is the first infinite ordinal, there exists $\beta \leq \omega$ such that

$$
\begin{aligned}
U_{\phi}=\left(-a_{0}, a_{0}\right) \cup\left\{\bigcup_{n<\beta}\left\{\left(-b_{n},-a_{n}\right) \cup\left(a_{n}, b_{n}\right)\right\}\right\} & \\
\qquad 0 \leq a_{0} \leq a_{n}<b_{n}, n \geq 1, &
\end{aligned}
$$

where $a_{0}=\sup \{u \geq 0: \phi(u)=0\}$ and $\left\{\left(a_{n}, b_{n}\right)\right\}_{n<\beta}$ is the countable family of intervals of $\mathbf{R}^{+}$where $\phi$ is affine. We put $m_{n}$ for the gradient of $\phi$ on $\left(a_{n}, b_{n}\right), n \geq 1$.

If $X$ is a Banach lattice, an ideal of $X$ is a closed linear subspace $Y \subseteq X$ such that if $x \in X, y \in Y$ and $|x| \leq|y|$, then $x \in Y$. In the following we use the notation $x \vee y=\sup \{x, y\}, x \wedge y=\inf \{x, y\}$ and $\chi_{A}$ for the characteristic function of the set $A$.

1. Lemma. Let $(\Omega, \Sigma, \mu)$ be a measure space, $L^{\phi}(\mu)$ an Orlicz space and $Y \subseteq L^{\phi}(\mu)$ an ideal. Then:

(I) If $f \in Y$ and $A \in \Sigma$, we have $\chi_{A} \cdot f \in Y$ and the function $G: Y \rightarrow Y$ such that $G(f)=\chi_{A} \cdot f, f \in Y$, is continuous.

(II) Let $c, d: \Omega \rightarrow \mathbf{R}$ be measurable functions and $h \in Y, 0 \leq h$. For each $g \in L^{\phi}(\mu)$ define $F(g)=h \wedge[0 \vee[(d-g) \wedge(g-c)]]$. Then $F(g) \in Y$ and $F: L^{\phi}(\mu) \rightarrow Y$ is continuous.

Proof. (I). Since $\left|\chi_{A} \cdot f\right| \leq|f|$, clearly $\chi_{A} \cdot f \in Y$. To prove continuity, observe that, for $f, g \in Y,\left|\chi_{A} \cdot f-\chi_{A} \cdot g\right| \leq|f-g|$. So $\left\|\chi_{A} \cdot f-\chi_{A} \cdot g\right\| \leq\|f-g\|$.

(II). By definition of the function $F, F(g)$ is measurable and $0 \leq F(g) \leq h$, for each $g \in L^{\phi}(\mu)$. Hence $F(g) \in Y$. Put

$$
H\left(g_{1}, g_{2}\right)=h \wedge\left(d-g_{1}\right) \wedge\left(g_{2}-c\right), \quad g_{1}, g_{2} \in L^{\phi}(\mu) .
$$

As real functions, we have (see $[5$, p. 1])

$$
\left|H\left(g_{1}, g_{2}\right)-H\left(g_{2}, g_{2}\right)\right| \leq\left|g_{1}-g_{2}\right| \leq\left|H\left(g_{1}, g_{2}\right)-H\left(g_{1}, g_{1}\right)\right| .
$$

Then $\left|H\left(g_{1}, g_{1}\right)-H\left(g_{2}, g_{2}\right)\right| \leq 2\left|g_{1}-g_{2}\right|$. Therefore, for $g_{1}, g_{2} \in L^{\phi}(\mu)$,

$$
\begin{aligned}
\left|F\left(g_{1}\right)-F\left(g_{2}\right)\right| & =\left|0 \vee H\left(g_{1}, g_{1}\right)-0 \vee H\left(g_{2}, g_{2}\right)\right| \\
& \leq\left|H\left(g_{1}, g_{1}\right)-H\left(g_{2}, g_{2}\right)\right| \leq 2\left|g_{1}-g_{2}\right| .
\end{aligned}
$$

Thus $\left\|F\left(g_{1}\right)-F\left(g_{2}\right)\right\| \leq 2\left\|g_{1}-g_{2}\right\|$ which implies continuity of $F$. 
2. Lemma. Let $L^{\phi}(\mu)$ be an Orlicz space, $A \in \Sigma$ and define (where it exists) $F_{A}(f)=\int_{A} f d \mu$, for each $f \in L^{\phi}(\mu)$. Then:

(1) If $\mu(A)<+\infty, F_{A} \in\left(L^{\phi}(\mu)\right)^{*}\left(=\right.$ dual of $\left.L^{\phi}(\mu)\right)$.

(2) If $A=\bigcup_{n \geq 1} A_{n}$, disjoint partition, with $\mu\left(A_{n}\right)<\infty, n \geq 1$, and there exists a measurable function $g: \Omega \rightarrow \mathbf{R}, g \geq 0$, such that $\int_{A} g d \mu<$ $+\infty$, then $F_{A}$ is continuous on the set $\left\{f \in L^{\phi}(\mu):-g \leq f \leq g\right\}$.

Proof. (1). This result is true in Köthe functions spaces. So it is true for Orlicz spaces (see [5, p. 28])

(2). This follows immediately from (1).

3. Proposition. Let $L^{\phi}(\mu)$ be an Orlicz space and $X \subseteq L^{\phi}(\mu)$ an ideal such that, for each $f \in X \backslash\{0\}, I_{\phi}(f /\|f\|)=1$. Then $B_{X}$ is stable.

Proof. Take $f_{i} \in B_{X}, i=0,1,2$, with $f_{0}=\frac{1}{2}\left(f_{1}+f_{2}\right)$ and $V\left(f_{i}\right)$ a neighborhood of $f_{i}, i=1,2$, in $B_{X}$. We prove that $2^{-1}\left(V\left(f_{1}\right)+V\left(f_{2}\right)\right)$ is a neighborhood of $f_{0}$ in $B_{X}$.

Suppose that $\left\|f_{0}\right\|<1$. In this case, the following discussion is valid for any Banach space. As $\left\|f_{0}\right\|<1$, we can choose $\varepsilon>0$ and $g_{i} \in V\left(f_{i}\right), i=1,2$, such that $B\left(g_{i} ; \varepsilon\right) \subseteq V\left(f_{i}\right)$ and $2^{-1}\left(g_{1}+g_{2}\right)=f_{0}$. Hence

$$
B\left(f_{0} ; \varepsilon\right)=2^{-1}\left(B\left(g_{1} ; \varepsilon\right)+B\left(g_{2} ; \varepsilon\right)\right) \subseteq 2^{-1}\left(V\left(f_{1}\right)+V\left(f_{2}\right)\right)
$$

Therefore $2^{-1}\left(V\left(f_{1}\right)+V\left(f_{2}\right)\right)$ is a neighborhood of $f_{0}$ in $B_{X}$.

Assume now that $\left\|f_{0}\right\|=1$. In this case it is enough to find a continuous function $S=\left(S_{1}, S_{2}\right): B_{X} \rightarrow B_{X} \times B_{X}$ such that $S_{i}\left(f_{0}\right)=f_{i}, i=1,2$, and $\Phi_{1 / 2} \circ S=$ identity on $B_{X}, \Phi_{1 / 2}$ being the midpoint map $\Phi_{1 / 2}(u, v)=$ $2^{-1}(u+v)$. Indeed, $S^{-1}\left(V\left(f_{1}\right) \times V\left(f_{2}\right)\right)$ will be a neighborhood of $f_{0}$ in $B_{X}$ and $S^{-1}\left(V\left(f_{1}\right) \times V\left(f_{2}\right)\right) \subseteq 2^{-1}\left(V\left(f_{1}\right)+V\left(f_{2}\right)\right)$. For $w \in \Omega$ we have $\phi\left(f_{0}(w)\right) \leq 2^{-1}\left(\phi\left(f_{1}(w)\right)+\phi\left(f_{2}(w)\right)\right)$ a.e. because $\phi$ is convex. Since $\left\|f_{0}\right\|=1$, then $\left\|f_{1}\right\|=1=\left\|f_{2}\right\|$. From

$$
1=I_{\phi}\left(f_{0}\right) \leq 2^{-1}\left(I_{\phi}\left(f_{1}\right)+I_{\phi}\left(f_{2}\right)\right)=1,
$$

we obtain that $\phi\left(f_{0}(w)\right)=2^{-1}\left(\phi\left(f_{1}(w)\right)+\phi\left(f_{2}(w)\right)\right)$ a.e., that is, either $f_{0}(w)=$ $f_{i}(w)$ or $f_{0}(w)$ and $f_{i}(w), i=1,2$, belong to the closure of the same connected component of $U_{\phi}$ a.e. Put

$$
\begin{gathered}
A^{+}=\left\{w \in \Omega: f_{1}(w)>f_{0}(w), f_{0}(w) \in\left(-a_{0}, a_{0}\right)\right\} \\
A^{-}=\left\{w \in \Omega: f_{1}(w)<f_{0}(w), f_{0}(w) \in\left(-a_{0}, a_{0}\right)\right\}, \quad A=A^{+} \cup A^{-}
\end{gathered}
$$


and, for $n \geq 1$,

$$
\begin{aligned}
& U_{n}^{+}=\left\{w \in \Omega: f_{1}(w)>f_{0}(w), f_{0}(w) \in\left(a_{n}, b_{n}\right)\right\}, \quad U^{+}=\bigcup_{n \geq 1} U_{n}^{+} \\
& U_{n}^{-}=\left\{w \in \Omega: f_{1}(w)<f_{0}(w), f_{0}(w) \in\left(-b_{n},-a_{n}\right)\right\}, \\
& U^{-}=\bigcup_{n \geq 1} U_{n}^{-}, \quad U=U^{+} \cup U^{-} \\
& L_{n}^{+}=\left\{w \in \Omega: f_{1}(w)<f_{0}(w), f_{0}(w) \in\left(a_{n}, b_{n}\right)\right\}, \quad L^{+}=\bigcup_{n \geq 1} L_{n}^{+} \\
& L_{n}^{-}=\left\{w \in \Omega: f_{1}(w)>f_{0}(w), f_{0}(w) \in\left(-b_{n},-a_{n}\right)\right\}, \\
& L^{-}=\bigcup_{n \geq 1} L_{n}^{-}, L=L^{+} \cup L^{-} \\
& S_{n}=U_{n}^{+} \cup L_{n}^{+}, \\
& T_{n}=L_{n}^{-} \cup U_{n}^{-}
\end{aligned}
$$

Define functions $d, c: \Omega \rightarrow \mathbf{R}:$

$$
\begin{aligned}
& d=a_{0} \cdot \chi_{A}+\sum_{n \geq 1} b_{n} \cdot \chi_{S_{n}}+\sum_{n \geq 1}\left(-a_{n}\right) \cdot \chi_{T_{n}} \\
& c=-a_{0} \cdot \chi_{A}+\sum_{n \geq 1} a_{n} \cdot \chi_{S_{n}}+\sum_{n \geq 1}\left(-b_{n}\right) \cdot \chi_{T_{n}}
\end{aligned}
$$

and, for each $g \in X$, put $F(g)=\left|f_{1}-f_{0}\right| \wedge[0 \vee[[d-g] \wedge[g-c]]]$. Observe that $F\left(f_{0}\right)=f_{1}-f_{0}$ on $U^{+} \cup A^{+} \cup L^{-}, F\left(f_{0}\right)=f_{0}-f_{1}$ on $U^{-} \cup L^{+} \cup A^{-}$and $F\left(f_{0}\right)=0$ outside of these sets. By 1 . Lemma, $F: X \rightarrow X$ is continuous just as functions $R_{i}, i=1,2$, defined by

$$
g \in X, \quad R_{i}(g)=g-(-1)^{i} \cdot\left[\chi_{U^{+} \cup A^{+} \cup L^{-}}-\chi_{U^{-} \cup A^{-} \cup L^{+}}\right] \cdot F(g) .
$$

Also we have $R_{i}\left(f_{0}\right)=f_{i}, i=1,2$, and $g=2^{-1}\left(R_{1}(g)+R_{2}(g)\right)$, for each $g \in X$. To get that $R_{i}(g) \in B_{X}$ if $g \in B_{X}$, we must modify $R_{i}$. Define $P, Q: B_{X} \rightarrow \mathbf{R}$ by

$$
\begin{array}{r}
g \in B_{X}, \quad P(g)=\int_{U}\left[\phi\left(R_{1}(g)\right)-\phi(g)\right] d \mu, \\
Q(g)=\int_{L}\left[\phi(g)-\phi\left(R_{1}(g)\right)\right] d \mu .
\end{array}
$$

We claim that $P, Q$ are continuous on $B_{X}$. We study function $P$; function $Q$ is similar. For $g \in B_{X}$ and $n \geq 1$, write $U_{n}^{+}(g)=\chi_{L_{n}^{+}} \cdot F(g)$ and $U_{n}^{-}(g)=$ $\chi_{U_{n}^{-}} \cdot F(g)$. Note that, on $U_{n}^{+} \cup U_{n}^{-}$,

$$
\begin{aligned}
0 & \leq \phi\left[R_{1}(g)\right]-\phi(g)=\phi\left[g+U_{n}^{+}(g)-U_{n}^{-}(g)\right]-\phi(g)=m_{n} \cdot F(g) \\
& \leq m_{n} \cdot\left|f_{1}-f_{0}\right|=\phi\left(f_{1}\right)-\phi\left(f_{0}\right),
\end{aligned}
$$


where $m_{n}$ is the gradient of $\phi$ in $\left(a_{n}, b_{n}\right)$. Also $0 \leq \phi\left(f_{1}\right)-\phi\left(f_{0}\right)$ on $U$ and $\int_{U}\left[\phi\left(f_{1}\right)-\phi\left(f_{0}\right)\right] d \mu<\infty$. So continuity of $P$ follows from the continuity of the function

$$
g \rightarrow \int_{U_{n}^{+} \cup U_{n}^{-}} F(g) d \mu, \quad g \in B_{X},
$$

and to prove this continuity it is enough, by 2 . Lemma, to prove that $U_{n}^{+} \cup U_{n}^{-}$ is $\sigma$-finite. Suppose that $\mu\left(U_{n}^{-}\right)=+\infty$. Then necessarily $\phi\left(a_{n}\right)=0$, since, if $\phi\left(a_{n}\right)>0$, we would obtain

$$
\int_{\Omega} \phi\left(f_{0}\right) d \mu \geq \int_{U_{n}^{+}} \phi\left(f_{0}\right) d \mu \geq \int_{U_{n}^{+}} \phi\left(a_{n}\right) d \mu=+\infty .
$$

Take a decreasing real sequence $\left\{t_{k}\right\}_{k \geq 1}$ such that $a_{n}<t_{k}<b_{n}, t_{k} \downarrow a_{n}$, and put $H_{k}=\left\{w \in U_{n}^{+}: f_{0}(w) \in\left[t_{k}, b_{n}\right)\right\}, k=1,2, \ldots$. Then $U_{n}^{+}=\bigcup_{k \geq 1} H_{k}$ and $\mu\left(H_{k}\right)<+\infty$. Analogously, $U_{n}^{-}$is also $\sigma$-finite.

Since $R_{i}\left(f_{0}\right)=f_{i}, i=1,2$, we have that $P\left(f_{0}\right)=Q\left(f_{0}\right)$. If $P\left(f_{0}\right)=0$, then $\mu(U)=0=\mu(L)$ and, for each $g \in B_{X}, P(g)=0=Q(g)$. Hence $I_{\phi}\left(R_{i}(g)\right)=I_{\phi}(g) \leq 1$ and $R_{i}(g) \in B_{X}, i=1,2$. So function $S=\left(R_{1}, R_{2}\right)$ holds.

Suppose that $P\left(f_{0}\right)>0$ and let $V\left(f_{0}\right)$ be the neighborhood

$$
V\left(f_{0}\right)=\left\{g \in B_{X}: P(g)>2^{-1} P\left(f_{0}\right)<Q(g)\right\}
$$

and $\psi: B_{X} \rightarrow[0,1]$ a continuous function such that $\psi(g)=0$, if $g \in$ $B_{X} \backslash V\left(f_{0}\right)$, and $\psi\left(f_{0}\right)=1$. Define $S_{i}(g), g \in B_{X}, i=1,2$, by

$$
\begin{aligned}
S_{i}(g)=g-(-1)^{i} \psi(g)[ & \min \left[\frac{Q(g)}{P(g)}, 1\right]\left(\chi_{U^{+} \cup A^{+}}-\chi_{U^{-}}\right) \\
& \left.-\min \left[\frac{P(g)}{Q(g)}, 1\right]\left(\chi_{L^{+} \cup A^{-}}-\chi_{L^{-}}\right)\right] \cdot F(g)
\end{aligned}
$$

Observe that:

(1) $S=\left(S_{1}, S_{2}\right)$ is continuous, $S_{i}\left(f_{0}\right)=f_{i}, i=1,2$, and, for each $g \in B_{X}$, $2^{-1}\left[S_{1}(g)+S_{2}(g)\right]=g$.

(2) If $g \in B_{X}$, then $I_{\phi}(g)=I_{\phi}\left(S_{i}(g)\right), i=1,2$, so that $S_{i}(g) \in B_{X}$. Indeed, if $g \notin V\left(f_{0}\right)$, it is clear because $S_{i}(g)=g$ in this case. Suppose that $g \in V\left(f_{0}\right)$ and, for example, that $P(g) \geq Q(g)$. Then

$$
\begin{aligned}
\int_{\Omega} \phi\left(S_{1}(g)\right) d \mu= & \int_{\Omega} \phi(g) d \mu \\
& +\int_{U}\left\{\phi\left[g+\psi(g) \cdot \frac{Q(g)}{P(g)}\left(\chi_{U^{+}}-\chi_{U^{-}}\right) F(g)\right]-\phi(g)\right\} d \mu \\
& +\int_{L}\left\{\phi\left[g-\psi(g)\left(\chi_{L^{+}}-\chi_{L^{-}}\right) F(g)\right]-\phi(g)\right\} d \mu \\
= & \int_{\Omega} \phi(g) d \mu+\psi(g) \cdot \frac{Q(g)}{P(g)} \cdot P(g)-\psi(g) \cdot Q(g)=\int_{\Omega} \phi(g) d \mu
\end{aligned}
$$

Analogously, $I_{\phi}\left(S_{2}(g)\right)=I_{\phi}(g)$. 
4. Remark. Note that in 3. Proposition it is proved that if $L^{\phi}(\mu)$ is an Orlicz space and $f_{i} \in B_{L} \phi_{(\mu)}, i=0,1,2, f_{0}=2^{-1}\left(f_{1}+f_{2}\right)$, and $V\left(f_{1}\right), V\left(f_{2}\right)$ are neighborhoods of $f_{1}, f_{2}$, respectively, in $B_{L}^{\phi}(\mu)$, then $2^{-1}\left(V\left(f_{1}\right)+V\left(f_{2}\right)\right)$ is a neighborhood of $f_{0}$ when $\left\|f_{0}\right\|<1$ or $I_{\phi}\left(f_{0}\right)=1$.

5. Corollary. Let $L^{\phi}(\mu)$ be an Orlicz space.

(1) If $E^{\phi}$ is the subspace of finite elements of $L^{\phi}(\mu)$, that is, $E^{\phi}=\{f \in$ $L^{\phi}(\mu): I_{\phi}(\lambda f)<\infty$, for each $\left.\lambda>0\right\}$, then $B_{E} \phi$ is stable.

(2) If $\phi$ verifies condition $\left(\Delta_{2}\right)$ or $\left(\delta_{2}\right)$, depending on $\mu$ (see [7], 8.14. Theorem, $p$. 53), so that $E^{\phi}=L^{\phi}(\mu)$, then $B_{L^{\prime \prime}(\mu)}$ is stable.

Proof. The subspace $E^{\phi}$ is an ideal of $L^{\phi}(\mu)$ such that $I_{\phi}(f /\|f\|)=1$ for each $f \in E^{\phi} \backslash\{0\}$. Now apply 3. Proposition.

6. Corollary. Let $L^{\phi}(\mu)$ be an Orlicz space and $X \subseteq L^{\phi}(\mu)$ an ideal such that $I_{\phi}(f /\|f\|)=1$, for each $f \in X \backslash\{0\}$. Then:

(1) If $K$ is a compact Hausdorff and $f \in C(K, X)$, the following are equivalent: (a) $f \in$ Ext. $B_{C(K, X)}$; (b) $f(k) \in$ Ext. $B_{X}$ for each $k \in K$.

(2) If $K$ is a Choquet simplex (see [1, p. 84]) and $f \in A(K, X) \quad(=$ Banach space of affine continuous functions $g: K \rightarrow X)$, the following are equivalent: (a) $f \in$ Ext. $B_{A(K, X)}$; (b) $f(k) \in$ Ext. $B_{X}$, for each $k \in$ Ext. $K$.

Proof. (1) $(a \Rightarrow b)$. Since $B_{X}$ is stable, the map $x \rightarrow\left\{z \in B_{X}\right.$ : there exists $y \in B_{X}$ with $\left.2^{-1}(z+y)=x\right\}$ is lower semicontinuous on $B_{X}$. Hence, applying the Michael's selection theorem [6], if $f(k) \notin$ Ext. $B_{X}$ for some $k \in K$, we can represent $f$ as a nontrivial convex combination $f=2^{-1}(g+h), g, h \in$ $B_{C(K, X)}$.

$(b \Rightarrow a)$. This follows immediately.

(2). This is consequence of 2.1. Theorem [2].

7. Remark. Suppose that $L^{\phi}(\mu)$ is an Orlicz space such that $B_{L^{\prime \prime}(\mu)}$ is stable. Does this condition imply that $E^{\phi}=L^{\phi}(\mu)$ ? If $(\Omega, \Sigma, \mu)$ has some infinite atom and $a_{0}=\sup \{t \geq 0: \phi(t)=0\}$ satisfies $a_{0}>0$, then always $E^{\phi} \neq L^{\phi}(\mu)$ but $B_{L^{\prime \prime}(\mu)}$ might be stable. For instance, if $(\Omega, \Sigma, \mu)$ reduces to a family $\left\{T_{i}\right\}_{i \in I}$ of infinite atoms, then $E^{\phi}=\{0\}$ and $L^{\phi}(\mu)$ is isometric to $\ell^{\infty}(I)$, whose closed unit ball $B_{f^{\infty}(I)}$ is stable because $\ell^{\infty}(I)$ is 3.2.I.P.

But if $\phi$ is strictly convex, then $E^{\phi}=L^{\phi}(\mu)$ and $B_{L^{\prime \prime}(\mu)}$ stable are equivalent, as is proved below. We need the following well-known Lemma.

8. Lemma. Let $(\Omega, \Sigma, \mu)$ be a measure space and $\phi$ a strictly convex Orlicz function. Then for $e \in L^{\phi}(\mu)$ the following are equivalent:

(1) $e \in$ Ext. $B_{L^{\prime \prime}(\mu)}$;

(2) $I_{\phi}(e)=1$ 
Proof. $(1 \Rightarrow 2)$. Suppose $I_{\phi}(e) \leq 1-\varepsilon, 0<\varepsilon \leq 1$. We can find $0<t_{0}<\infty$ and $A \in \Sigma, A \subseteq\left\{w \in \Omega:|e(w)| \leq t_{0}\right\}$ such that $0<\mu(A)<+\infty$. Choose $\delta>0$ verifying $\int_{A} \phi(e \pm \delta) d \mu \leq \int_{A} \phi(e) d \mu+\varepsilon$ and define $e_{1}=e+\delta \cdot \chi_{A}$, $e_{2}=e-\delta \cdot \chi_{A}$. Then clearly $\left\|e_{i}\right\| \leq 1, e=\frac{1}{2}\left(e_{1}+e_{2}\right)$ but $e_{1} \neq e \neq e_{2}$. Therefore $I_{\phi}(e)=1$.

$(2 \Rightarrow 1)$. Since $I_{\phi}(e)=1,\|e\|=1$. Assume that $e=\frac{1}{2}\left(e_{1}+e_{2}\right),\left\|e_{i}\right\|=1$, $i=1,2$. Since $I_{\phi}$ and $\phi$ are convex and $I_{\phi}\left(e_{i}\right) \leq 1$, we obtain that $I_{\phi}\left(e_{i}\right)=1$, $i=1,2$, and $\phi(e(w))=\frac{1}{2}\left(\phi\left(e_{1}(w)\right)+\phi\left(e_{2}(w)\right)\right), w \in \Omega$, a.e. But $\phi$ is strictly convex. Therefore $e(w)=e_{1}(w)=e_{2}(w)$ a.e. and $e=e_{1}=e_{2}$.

9. Proposition. Let $\phi$ be strictly convex and $(\Omega, \Sigma, \mu)$ a measure space. Then the following are equivalent:
(1) $B_{L^{\phi}(\mu)}$ is stable;
(2) $E^{\phi}=L^{\phi}(\mu)$.

Proof. $(2 \Rightarrow 1)$. This is 3 . Proposition.

$(1 \Rightarrow 2)$. First recall that, if $f \in L^{\phi}(\mu)$ and $I_{\phi}(f)<1$, the following are equivalent: (i) $\|f\|=1$; (ii) $I_{\phi}(\lambda f)=+\infty$ for each $\lambda>1$. Suppose that $E^{\phi} \neq$ $L^{\phi}(\mu)$. We claim that there exists $f \in S_{L} \phi_{(\mu)}$ such that $I_{\phi}(f)<1$. Indeed, as $E^{\phi} \neq L^{\phi}(\mu)$, there exist $g \in S_{L} \phi_{(\mu)}$ and $\lambda>1$ such that $I_{\phi}(\lambda g)=\infty$. Let $a=\inf \left\{\lambda>0: I_{\phi}(\lambda g)=\infty\right\}$. Clearly $a \geq 1$ because, as $g \in B_{L^{\prime \prime}(\mu)}, I_{\phi}(g) \leq 1$. If $A=\{w \in \Omega: g(w) \neq 0\}$, take a disjoint partition $A=\bigcup_{n \geq 1} A_{n}$ such that $\mu\left(A_{n}\right)<\infty$ and $g$ bounded on $A_{n}, n \geq 1$. Now $\int_{A_{n}} \phi(\lambda g) d \bar{\mu}<\infty$ for each $\lambda>0$ and $n \geq 1$. Since $I_{\phi}\left(\left(a-2^{-k}\right) g\right)<\infty$ but $I_{\phi}\left(\left(a+2^{-k}\right) g\right)=\infty, k \in \mathbf{N}$, we can choose a sequence $\left\{N_{k}\right\}_{k \geq 1}$ of finite subsets of $\mathbf{N}$ verifying:

(a) If $k_{1}<k_{2}, n<m$ for each $n \in N_{k_{1}}, m \in N_{k_{2}}$.

(b) $\sum_{n \in N_{k}} \int_{A_{n}} \phi\left(\left(a-2^{-k}\right) g\right) d \mu \leq 2^{-(k+1)}$.

(c) $\sum_{n \in N_{k}} \int_{A_{n}} \phi\left(\left(a+2^{-k}\right) g\right) d \mu \geq k$.

Define $f(w)=\left(a-2^{-k}\right) g(w)$ if $w \in A_{n}$ and $n \in N_{k}$ and $f(w)=0$ if $w \notin\left(\bigcup_{k \geq 1} \bigcup_{n \in N_{k}} A_{n}\right)$. Then $I_{\phi}(f)<1$ but $I_{\phi}(\lambda f)=\infty$ for each $\lambda>1$. Thus $\|f\|=1$.

Take this function $f$ and $\varepsilon>0$ arbitrarily. We prove that there exists $g \in$ Ext. $B_{L^{\circ}(\mu)}$ verifying $\|f-g\| \leq \varepsilon$. Since $I_{\phi}((1+\varepsilon) f)=+\infty$, there exists $B \in \Sigma, 0<\mu(B)<\infty$, such that $\int_{B} \phi((1+\varepsilon) f) d \mu \geq 1-I_{\phi}(f)$. So there exists $0<\eta \leq \varepsilon$, verifying

$$
\int_{\Omega \backslash B} \phi(f) d \mu+\int_{B} \phi((1+\eta) f) d \mu=1 .
$$

Define $g(w)=f(w)$ if $w \in \Omega \backslash B$ and $g(w)=(1+\eta) f(w)$ if $w \in B$. Then, by the above 8 . Lemma, $g \in$ Ext. $B_{L^{\prime \prime}(\mu)}$ and, as $|f(w)-g(w)| \leq|\varepsilon \cdot f(w)|$, 
also $\|f-g\| \leq \varepsilon$. With $\varepsilon$ arbitrary, we conclude that $f$ belongs to the closure of Ext. $B_{L^{\phi}(\mu)}$. But Ext. $B_{L^{\phi}(\mu)}$ is closed because $B_{L^{\phi}(\mu)}$ is stable. Since clearly $f \notin$ Ext. $B_{L^{\phi}(\mu)}$, we obtain a contradiction.

\section{REFERENCES}

1. E. M. Alfsen, Compact convex sets and boundary integrals, Springer-Verlag, 1971.

2. A. Clausing and S. Papadopoulou, Stable convex sets and extremal operators, Math. Ann. 231 (1978), 193-203.

3. R. Grzaslewicz, Finite dimensional Orlicz spaces, Bull. Polish Acad. Sci. Math. 33 (1985), no. 5-6, 277-283.

4. _ـ Extremes points in $C\left(K, L^{\phi}(\mu)\right)$, Proc. Amer. Math. Soc. 98 (1986), 611-614.

5. J. Lindenstrauss and L. Tzafriri, Classical Banach spaces II, Springer-Verlag (1979).

6. E. Michael, Continuous selections I, Ann. of Math. 63 (1956), 361-382.

7. J. Musielak, Orlicz spaces and modular spaces, Lecture, Notes in Math., vol. 1034, 1983, Springer-Verlag.

8. S. Papadopoulou, On the geometry of stable compact convex sets, Math. Ann. 229 (1977), 193-200.

Facultad de Matemáticas, $D^{\text {to }}$ De Análisis Matemático, Universidad Complutense de MADRID, 28040-MADRID, SPAIN 